\title{
Klaus Hasselmann, la mer comme laboratoire
}

Comme l'écrit le comité Nobel ${ }^{1}$ : Klaus Hasselmann was creatively using fundamental physics concepts to quantify the surface ocean wave spectra, thereby building a deep appreciation for the nature of fluctuations on the sea-surface. Building on the intuition garnered from this research, basic concepts in turbulence and Lorenz's chaotic weather, he derived a generalizable stochastic description of ocean climate in which the "noise" is associated with the "weather" as described above. His work has provided both the motivation and the observational structure for climate scientists to address variability.

Qu'on peut traduire ainsi : « Klaus Hasselmann utilisait de manière créative des concepts de physique fondamentale pour quantifier le spectre des vagues océaniques de surface, élaborant ainsi une appréciation approfondie de la nature des fluctuations à la surface de la mer. S'appuyant sur l'intuition qu'il avait recueillie lors de cette recherche, sur les concepts de base en turbulence et sur le caractère chaotique de la météo mis en évidence par Lorenz, il a tiré une description stochastique généralisable du climat de l'océan dans laquelle le « bruit » est l'analogique de « la météo » tel que décrit ci-dessus. Son travail a fourni aux climatologues à la fois la motivation et la démarche d'analyse des observations pour aborder la variabilité. »

La première partie de ce paragraphe a un écho profond pour tous ceux qui comme moi ont connu et travaillé avec Klaus Hasselmann sur les vagues océaniques et qui savent l'importance qu'il y accordait. Cet intérêt s'est ancré solidement dès les débuts de sa carrière. Son premier poste, de 1957 à 1961, à l'Institut d'architecture navale de l'université de Hambourg, comme assistant de recherche de Karl Wieghardt, un élève et collaborateur de Prandtl (Eckert, 2017), et ses premières publications en attestent. La liste chronologique de ses publications sur le site de l'Academia Europaea ${ }^{2}$, fait état dès 1960 d'un article sur les équations de base de la prévision de l'état de la mer (Hasselmann, 1960), suivi d'un autre, l'année suivante, sur l'échange d'énergie non linéaire au sein d'un spectre de houle (Hasselmann, 1961). Ce sont les premiers d'une longue série de publications sur les vagues. Dès le début, on y retrouve sa vision ample et unificatrice entre théorie, modélisation et observation.

De cette époque date le déploiement des premiers instruments permettant une analyse spectrale des vagues océaniques et la découverte que leur distance à la source qui les avait créées pouvait être déduite du décalage successif des pics spectraux vers des fréquences plus élevées. Klaus Hasselmann a participé à l'une de ces premières campagnes de mesure (voir l'article dont il est co-auteur (Snodgrass et al., 1966), qui inclut un bref aperçu de l'état des connaissances à cette époque). Sa prochaine étape sera d'organiser, en Europe, une grande campagne de mesure. Ce sera le projet Jonswap (Joint North Sea Wave Project) de 1968 à 1969 (Hasselmann et al., 1973) qui permettra d'affiner l'observation des transferts non linéaires d'énergie au sein des vagues lors de leur croissance et de caractériser leur état de croissance par la forme de ce spectre. La paramétrisation de cette forme va aider à franchir une première étape pour la prise en compte des interactions non-linéaires dans la modélisation et la prévision numérique opérationnelle de l'état de la mer alors très contraintes par les capacités de calcul des ordinateurs (Hasselmann et al., 1976).

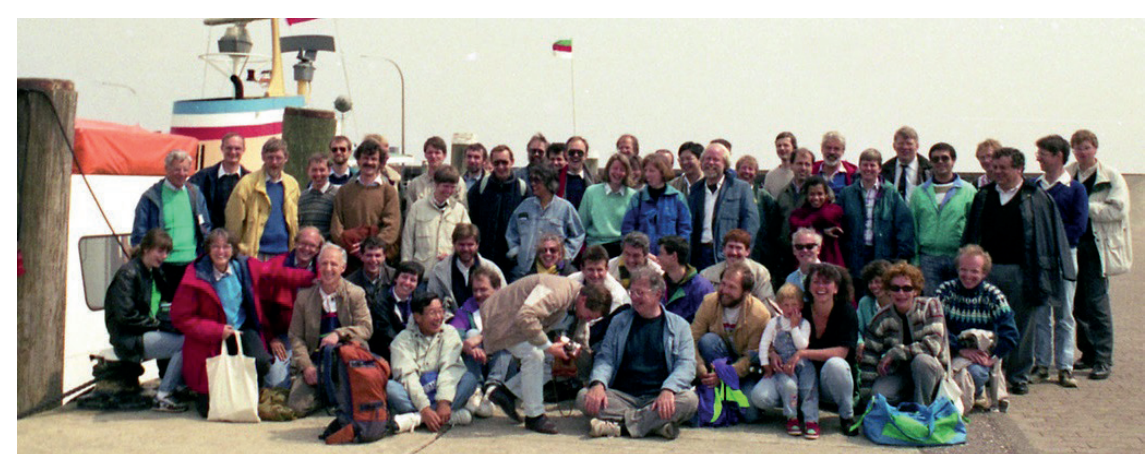

Klaus Hasselmann (debout, $8^{e}$ à partir de la droite) et Susanne Hasselmann (assise, $2^{e}$ à partir de la gauche) lors de la réunion du groupe WAM à Sylt en juin 1991. (C) Anne Guillaume.
Au début des années 1980, avec son épouse Susanne, mathématicienne et informaticienne, Klaus se lance dans le projet de développer un modèle qui utilise ses travaux théoriques sur les interactions non linéaires (Hasselmann et Hasselmann, 1985) pour mieux analyser et prévoir les vagues. Parallèlement il mobilise la communauté des modélisateurs dans une grande campagne de comparaison de leurs modèles de vagues (Allender et al., 1985). Des conclusions issues de cette campagne va naître le groupe WAM (Wave Modeling group) afin de développer un nouveau modèle qui prenne pleinement en compte les travaux de Klaus Hasselmann sur les interactions non linéaires. Rejoindre ce groupe en 1985 a eu pour moi une importance capitale. Cela a donné une autre dimension aux travaux que je poursuivais. La diversité des compétences - de la théorie, l'observation à la modélisation - et des nationalités qui s'y retrouvaient m'a enthousiasmée. Cela m'a permis de conforter la validité, au regard des contraintes opérationnelles existantes, des options que j'avais prises dans le nouveau modèle que Météo-France m'avait chargée de développer. Et surtout de rejoindre l'équipe qui œuvrait au développement du modèle WAM (Bauer et al., 1988), puis de me voir confier en 1992, par le Centre européen pour les prévisions météorologiques à moyen terme de Reading, la mise en place opérationnelle de ce modèle qui a eu lieu le $1^{\text {er }}$ juillet 1992. Nous avions tous conscience que Klaus Hasselmann était au cœur du déroulement de cette aventure, même si à l'époque les plus jeunes ne mesuraient ni toutes les facettes ni toute l'ampleur de son rôle. La réunion annuelle était le point d'orgue des collaborations : Bergen, Venise, Woods Hole, Paris (à Météo-France), Valencia, lac Couchiching au Canada, Sylt où s'était déroulée la campagne Jonswap, Sintra... Tous les membres du groupe s'y retrouvaient, Klaus et Susanne Hasselmann toujours, et bien d'autres qui n'auraient pour rien au monde manqué ces quelques jours d'échanges scientifiques, ni les moments festifs et conviviaux qui les accompagnaient. J'ai ainsi souvenir d'une soirée où nous étions tous autour d'un piano à

1. https://www.nobelprize.org/uploads/ 2021/10/sciback_fy_en_21.pdf

2. https://www.ae-info.org/ae/Member/ Hasselmann_Klaus/Publications 
chanter tandis que la lune se levait sur le lac Couchiching, de plusieurs promenades, à Woods Hole ou le long de la plage de Sylt, où le battement du ressac rythmait les discussions.

Il y aurait tant à dire, mais j'aimerais ici souligner un point pour lequel le rôle de Klaus Hasselmann a été déterminant et dont nous étions nombreux à avoir conscience : la construction d'un espace de projet européen. En Norvège, en Italie, en France, en Espagne, peut-être moins au Royaume-Uni, en Allemagne ou aux Pays-Bas, nous avions travaillé jusque-là dans des équipes petites et isolées. Le souffle de collaboration européenne que Klaus Hasselmann nous a communiqué dans l'application concrète de ses travaux pour la prévision opérationnelle des vagues océaniques a été déterminant. Il a fait de l'Europe le centre mondial de la modélisation des vagues et permis d'exporter le modèle WAM dans le monde entier. Qu'il en soit ici chaleureusement remercié.

\section{Anne Guillaume}

\footnotetext{
Pour en savoir plus :

Allender J.H., Barnett T.P., Bertotti L., Bruinsma J., Cardone V.J., Cavaleri L., Ephraums J., Golding B., Greenwood A., Guddal J., Günther H., Hasselmann K., Hasselmann S., Joseph P., Kawai S., Komen G.J., Lawson L., Linné H., Long R.B., Lybanon M., Maeland E., Rosenthal W., Toba Y., Uji T., de Voogt W.J.P., 1985. Ocean Wave Modeling, Part 1: The Sea Wave Modelling Project (SWAMP), Principal results and conclusions. Plenum Publishing Corporation.

Bauer E., Bertotti L., Cardone C.V., Ewing J.A., Greenwood J.A., Guillaume A., Hasselmann K., Hasselmann S., Janssen P.A.E.M., Komen G.J., Lionello P., Reistad M., Zambresky L., 1988. The WAM Model - A third generation ocean wave prediction model. J. Phys. Oceanogr., 18, 1775-1810.

Eckert M., 2017. Ludwig Prandtl and the growth of fluid mechanics in Germany. Comptes Rendus Mécanique, 345, 467-476. doi: 10.1016/j.crme. 2017.05.005

Hasselmann K., 1960. Grundgleichungen der Seegangsvoraussage. Schiffstechnik, 7, S. 191-195.

Hasselmann K., 1961. Über den nichtlinearen Energieaustausch innerhalb eines Seegangsspektrums. Sonderdruck aus Zeitschrift für Angewandte Mathematik und Mechanik, Sonderheft (GAMM-Tagung Würzburg), 41.

Hasselmann K., Barnett T.P., Bouws E., Carlson H., Cartwright D.E., Enke K., Ewing J.A., Gienapp H., Hasselmann D.E., Kruseman P., Meerburg A., Müller P., Olbers D.J., Richter K., Sell W., Walden H., 1973. Measurements of wind-wave growth and swell decay during the Joint North Sea Wave Project (JONSWAP). Ergänzungsheft zur Deutschen Hydrographischen Zeitschrift, Reihe A, 12.

Hasselmann K., Ross D.B., Müller P., Sell W., 1976. A parametric wave prediction model. J. Phys. Oceanogr., 6, 200-228.

Hasselmann S., Hasselmann K., 1985. Computations and parameterizations of the nonlinear energy transfer in a gravity wave spectrum. Part I: A new method for efficient computations of the exact nonlinear transfer integral. J. Phys. Oceanogr., 15, 1369-1377.

Snodgrass F.E., Groves G.W., Hasselmann K.F., Miller C.R., Munk W.H., Powers W.H., 1966. Propagation of ocean swell across the Pacific. Phil. Trans. R. Soc. A, 1103, 259, 431-497.
}

\section{Le PMIP fête ses 30 ans}

Depuis 30 ans, le Paleoclimate Modeling Intercomparison Project (PMIP) mobilise les groupes de modélisation et les spécialistes des reconstructions paléoclimatiques avec l'ambition de comprendre les variations climatiques passées et tester la capacité des modèles de climat à représenter un climat différent de l'actuel. Ce projet a été lancé en 1991 au cours d'un colloque international organisé en France par Jean Jouzel et Sylvie Joussaume. Cette mise en perspective à long terme reste toujours d'actualité au moment où le dernier rapport du Giec fait ressortir qu'il faut remonter plus de 2 millions d'années dans le passé pour retrouver les taux actuels de $\mathrm{CO}_{2}$ dans l'atmosphère, et que l'augmentation de la température moyenne du globe depuis la période préindustrielle excède les variations des 100000 dernières années (IPCC, 2021).

La spécificité du PMIP est de tester en conditions paléoclimatiques les modèles utilisés pour les projections futures. Ces modèles ont évolué à partir de modèles d'atmosphère vers des modèles complets du système Terre (figure 1). Néanmoins, la complexité

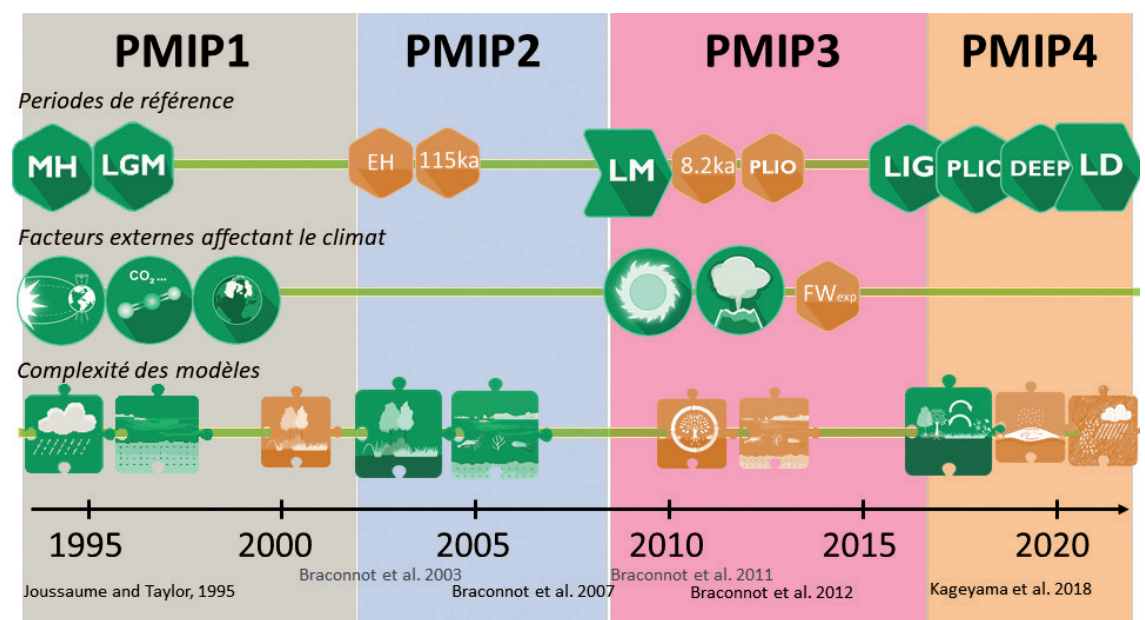

Figure 1. Frise montrant l'évolution du PMIP, en mettant l'accent sur les quatre phases, les périodes de référence considérées, les facteurs externes affectant le climat et la complexité des modèles. Les éléments en vert correspondent à ce qui est traité par l'ensemble des groupes participant au projet, alors que les éléments en orange indiquent des facteurs additionnels considérés par un sousensemble de participants. Les références sont les références clés des quatre phases, en noir dans des revues à comité de lecture et en bleu dans le magazine du programme Clivar du Programme mondial de recherche sur le climat (PMRC).

des modèles et le niveau d'intégration du cycle du carbone, de la dynamique de la végétation ou des aérosols varient encore fortement d'un modèle à l'autre. À chaque nouvelle phase du PMIP (figure 1), les ordinateurs crépitent dans plus de 20 pays répartis sur le continent américain, Europe et Asie, et les bases de données absorbent des pétaoctects bien formatés pour favoriser les échanges, ainsi que les comparaisons modèles-modèles et modèles-données. Sédiments marins, carottes de glace, pollen, niveaux passés des lacs, cernes 